\title{
A Multivariate Jump-Driven Financial Asset Model*
}

\author{
Elisa Luciano ${ }^{\dagger}$ and Wim Schoutens ${ }^{\ddagger}$
}

April 19, 2005

\begin{abstract}
In this paper, we propose a multivariate model for financial assets which incorporates jumps, skewness, kurtosis and stochastic volatility, and discuss its applications in the context of equity and credit risk. In the former case we describe the stochastic behavior of a series of stocks or indexes, in the latter we apply the model in a multi-firm, value-based default model.

Starting from a independent Brownian world, we will introduce jumps and other deviations from normality, as well as non-Gaussian dependence, by the simple but very strong technique of stochastic time-changing. We work out the details in the case of a Gamma time-change, thus obtaining a multivariate Variance Gamma (VG) setting.

We are able to characterize the model from an analytical point of view, by writing down the joint distribution function of the assets at any point in time and by studying their association via the copula technique.

The model is also computationally friendly, since numerical results require a modest amount of time and the number of parameters grows linearly with the number of assets. The main feature of the model however is the fact that - opposite to other, non jointly Gaussian settings - its risk neutral dependence can be calibrated from univariate derivative prices. Examples from the equity and credit market show the goodness of fit attained.
\end{abstract}

\section{Introduction}

The multivariate modelling of financial assets has become more and more important in modern finance. In many branches, such as equity, fixed income and credit risk, derivatives depending on a multiple of underlyers have found their way. Basket options on equities, First-to-Default (FtD) and Collateralized Debt Obligations $(\mathrm{CDO}), \mathrm{CDO}^{2}$ are typical examples of these multivariate structures. In modelling the underlyers, dependence plays a crucial role. Both at the marginal and joint level, one would like to move from the typical Brownian world into a more realistic setting, taking into account jumps, skewness, kurtosis, stochastic volatility, etc. However, the resulting model, in order to be useful in practice, should also remain tractable. This means that it should be possible to calibrate it to the relevant market and to compute the prices of the derivatives in a reasonable amount of time.

In this paper, we propose a multivariate model for financial assets which aims at satisfying these requirements and discuss its applications in the context of equity and credit risk. On the one side, we model the stochastic behavior of a series of stocks or indexes, on the other we apply the model in a firm-value based context to model default in a multivariate setting.

Starting from a independent Brownian world, we will introduce jumps, skewness, kurtosis, stochastic volatility and non Gaussian dependence by the simple but very strong technique of stochastic time-changing. We work out the details in case of a Gamma time-change, which brings us in a multivariate Variance Gamma (VG) setting. The VG model has already proven its power in a univariate setting: we explore its multivariate properties.

We will show how one can calibrate the model to a set of basic - and above all univariate - derivative instruments, namely vanilla options in the equity case and Credit Default Swaps in the credit setting. The

\footnotetext{
${ }^{*}$ This manuscript also appeared as UCS Technical Report 2005-02, U.C.S., K.U.Leuven and as ICER Working Paper 06/05, Turin.

$\dagger$ University of Turin \& ICER, Villa Gualino, V. S. Severo, 63 I-10133 Torino, Italy. E-mail: luciano@econ.unito.it

${ }^{\ddagger}$ K.U.Leuven, U.C.S., W. De Croylaan 54, B-3001 Leuven, Belgium. E-mail: Wim.Schoutens@wis.kuleuven.ac.be
} 
calibration on univariate derivatives is peculiar of this model and extremely helpful. Indeed, whenever the assumption of Girsanov's theorem are not satisfied, for instance when financial returns are assumed to be marginally but not jointly normally distributed, dependence under the historical and risk neutral measure do not coincide, and the calibration of the joint behavior is usually restricted to the historical measure. Calibration under the risk neutral probability would require the existence of actively traded multivariate derivatives. This is often not the case. The difficulty is circumvented by the model presented here.

\section{From an Independent Gaussian World to a Dependent Lévy World}

\subsection{Motivation}

The starting point of the model is the independent Black-Scholes world, where a financial asset is typically modelled by a geometric Brownian Motion. For stocks this model was developed in [3]; the same assumptions are underlying the default models by Merton [26], Black and Cox [4], Leland [16], Longstaff and Schwartz [18] and the CreditGrades ${ }^{\mathrm{TM}}$ approach of [8].

We start with recalling the univariate setting. In this model $A_{t}$, the price process of an asset - say a stock, an index or a firm-value - is modelled as:

$$
A_{t}=A_{0} \exp \left(\theta t+\sigma W_{t}\right), \quad t \geq 0 .
$$

There are several shortcomings of this model, which we briefly list below.

- Normal Distribution: log-returns are normally distributed, whereas it would be better to have a more flexible distribution, in order to take into account skewness and excess kurtosis, which are typically observed in the market. As concerns equities, this was one of the main reason for the consideration of Lévy processes (see [30]).

- Continuous Sample Paths: a Brownian motion has continuous sample paths. However prices are in reality driven by jumps. Release of new information, which is immediately absorbed by the market, leads to a jump in the price process.

- Extreme Events: the model is not able to give realistic probabilities of extreme events (crashes, defaults, ...). The underlying reason is that the Normal distribution has too light tails and that the model produces continuous sample paths. With a reasonable volatility parameter $\sigma$, the probability of a significant move, say more than 5 percent, is completely unrealistic [2]. The Brownian motion needs a substantial time to reach a low barrier, where in reality jumps can cause an almost immediate move over the barrier.

If one likes to do multivariate modelling, in order to describe $n$ dependent price processes $\left(A_{t}^{(1)}, \ldots, A_{t}^{(n)}\right)$, a natural way is to consider a vector of $n$ dependent Brownian Motions $\left(W_{t}^{(1)}, \ldots, W_{t}^{(n)}\right)$. The dependence is uniquely defined by the correlation matrix. Each individual price process is modelled by a geometric Brownian motion:

$$
A_{t}^{(i)}=A_{0} \exp \left(\theta_{i} t+\sigma_{i} W_{t}^{(i)}\right), \quad t \geq 0 .
$$
lems:

Besides the shortcomings in the univariate situation, the multivariate model has also some additional prob-

- Gaussian Dependence: the dependence between the assets is described by a Gaussian structure: however, there is evidence that joint normality is not realistic, mainly because it does not present tail dependency.

- Estimation of Correlation: typically, this is done on the basis of historical data, hence leading to an estimate of the historical dependence structure. For the Gaussian setting (under the assumptions of Girsanov's theorem) this dependence structure is also the risk neutral one. However, as soon as one introduces more sophisticated assumptions on the marginal models, such as stochastic volatility features, while preserving a Gaussian dependence structure, there is no guarantee any more that dependence under the risk neutral and the historical measure coincide (see for instance [9]). Moreover, as with all historical data, a sudden change into the regime has only a marginal effect in the historical estimate, whereas the effect on the risk-neutral one can be much more pronounced. 
- Quadratic Number of Parameters: the number of parameters grows quadratically in $n$ through the correlation matrix. However the available market data, say derivative instrument prices, are usually linear in $n$.

We will try to solve the above problems by the simple but very powerful technique of stochastic timechanging. We will build a model, with a non-Normal underlying distribution, with jumps, more realistic extreme events probabilities, and, last but not least, a non-Gaussian dependence structure. The model can be calibrated by using only the (liquid) standard derivatives available in the market (historical data are not needed) and the number of parameters only grows linearly in the number of assets.

We will start from the independent Gaussian case in which all assets are driven by a geometric Brownian motion; the Brownian motions involved are assumed to be independent of each other. In order to introduce dependence, we time-change all the asset price processes by a common time-change. The interpretation is that all firms operate in the same economic environment. A jump in the time-change leads to a jump in the price processes and hence all moves/jumps (small and big ones) occur simultaneously; however the jump-sizes are caused by the individual Brownian motions. We thus introduce a new business time in which the general market operates. This new business time can also be interpreted as a model for the information arrival. Taking into account that the market will not forget information, the amount of information only can not decrease. Morever, it seems reasonable that the amount of new information released should not be affected by the amount already released, in other words, the information process should have independent increments. Finally, one can also require that the increment only depends on the length of that period and hence is stationary. This leads use to a model where the information process is modelled by an non-decreasing process with stationary and independent increments, i.e. a subordinator (a special case of a Lévy process).

Here we opt to work with the Gamma time-change, for tractability reasons. However, other time-changes, like the more general Generalized Inverse Gaussian (GIG), can be used in the same manner. Using a Gammatime change, we enter the realm of Variance Gamma; the GIG time change for example would lead to the Generalized Hyperbolic Model.

\subsection{A Multivariate Variance Gamma Model}

Let $G=\left\{G_{t}, t \geq 0\right\}$ be a Gamma process, i.e. a process which starts at zero, has stationary and independent increments; increments over the time interval $[s, s+t]$ follow a gamma distribution. More precisely, we have that $G$ is a Lévy process (a subordinator) (see the Appendix), where the defining distribution of $G_{1}$ is a Gamma $(a, b)$ distribution with density function

$$
f_{\text {Gamma }}(x ; a, b)=\frac{b^{a}}{\Gamma(a)} x^{a-1} \exp (-x b), \quad x \geq 0
$$

and characteristic function given by

$$
\phi_{\text {Gamma }}(u ; a, b)=(1-\mathrm{i} u / b)^{-a} .
$$

Standard Lévy process theory (see for example [1], [28] or [30]) teaches us that increments over intervals of length $s$ then are Gamma $(a s, b)$ distributed. For obvious, normalization reasons, we will work with a Gamma process such that $E\left[G_{t}\right]=t$, which in terms of the parameters imply that $a=b$. Let us denote by $\nu$ the common value $1 / a=1 / b$.

We thus model the asset values by geometrical Brownian Motions time-changed by a common Gamma business time (stochastic clock):

$$
A_{t}^{(i)}=A_{0} \exp \left(\theta_{i} G_{t}+\sigma_{i} W_{G_{t}}^{(i)}\right), \quad t \geq 0 .
$$

Now, it is well known that a Brownian Motion with drift time-changed by a Gamma process leads to the Variance Gamma (VG) process. The class of Variance Gamma distributions was introduced by Madan and Seneta [22] in the late 1980s as a model for stock returns. There (and in [23] and [21]) the symmetric case $(\theta=0)$ was considered. In [20], the general case with skewness is treated. In equity and interest rate modelling the process has already proven its modelling capabilities. See for example [30]. 
More precisely, we will have that the $i$ th price process is the exponential of a VG-process:

$$
A_{t}^{(i)}=A_{0} \exp \left(X_{t}^{(i)}\right), \quad t \geq 0,
$$

where $X^{(i)}=\left\{X_{t}^{(i)}, t \geq 0\right\}$ is a VG process with parameters $\left(\sigma_{i}, \nu, \theta_{i}\right)$.

The variance, skewness and kurtosis of a VG-distribution with parameters $(\sigma, \nu, \theta)$ are given in the next table:

$$
\begin{array}{lc} 
& \mathbf{V G}(\sigma, \nu, \theta) \\
\text { mean } & \theta \\
\text { variance } & \sigma^{2}+\nu \theta^{2} \\
\text { skewness } & \theta \nu\left(3 \sigma^{2}+2 \nu \theta^{2}\right) /\left(\sigma^{2}+\nu \theta^{2}\right)^{3 / 2} \\
\text { kurtosis } & 3\left(1+2 \nu-\nu \sigma^{4}\left(\sigma^{2}+\nu \theta^{2}\right)^{-2}\right)
\end{array}
$$

One thus can see that the variance decomposes into an idiosyncratic component $\sigma^{2}$ (coming from the original Brownian Motion) and an exogenous component $\nu \theta^{2}$ (arising from the time-change); $\theta$ tells us how much a specific company is exposed to a kind of global market uncertainty $(\nu)$. This leads to the interpretation that the asset volatility decomposes in an idiosyncratic volatility on top of which one adds a global common volatility. Note also that skewness and kurtosis are affected by the companies own specific settings as well as by the global parameter $\nu$. In Figure 1, one sees the sample paths of two dependent VG processes.

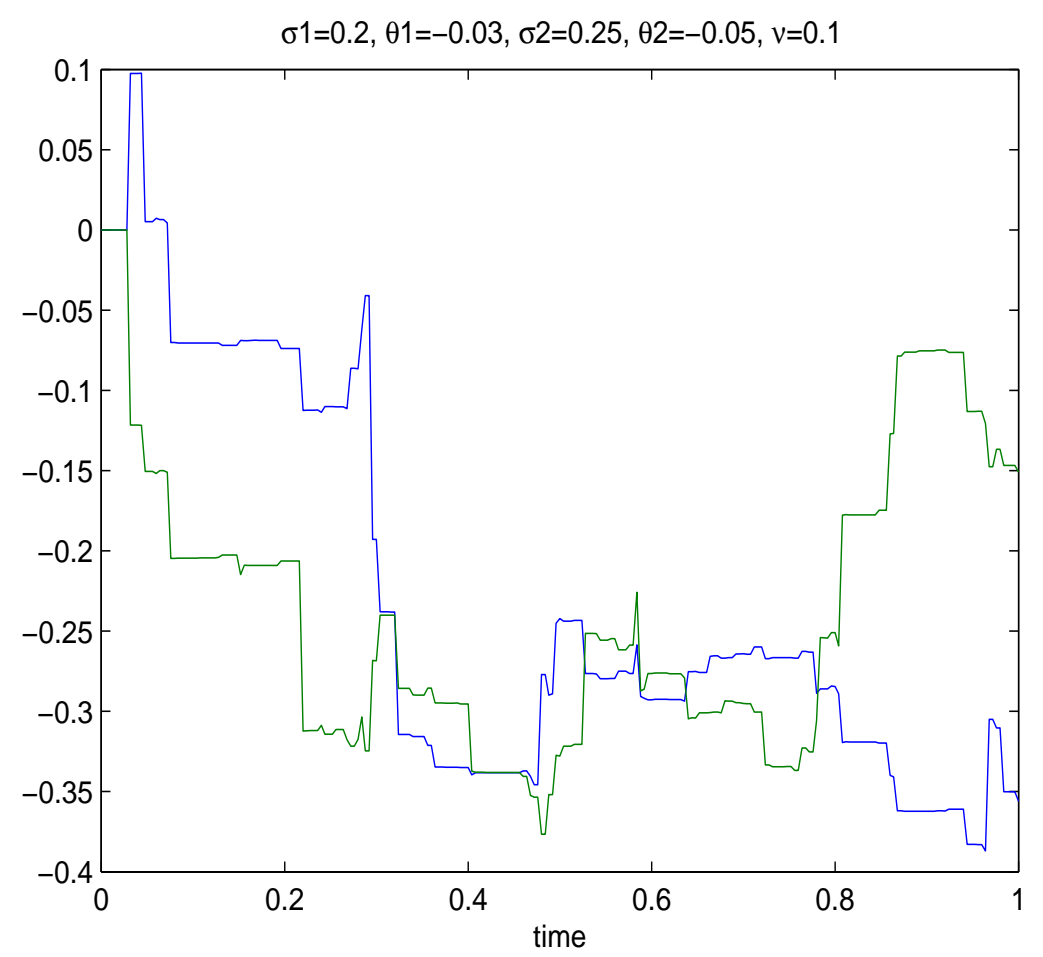

Figure 1: Sample path of correlated VG processes

\subsection{Risk Neutral Setting}

By the introduction of a new source of randomness with respect to Brownian motion (or equivalently by the introduction of jumps) the model belongs to the class of the incomplete models. For such models, there is not a unique equivalent martingale measure (EMM). For the pricing of derivatives and the calibration we will work in this paper with the mean-correcting EMM; the change of measure is completely analogous to the Black-Scholes 
setting, in which the (historical) mean parameter was changed into a new parameter in order to make the model risk-neutral. Here we do the same, we correct the process by shifting its mean value. More precisely, our risk-neutral dynamics for the asset prices are given by

$$
A_{t}^{(i)}=A_{0} \exp \left(\left(r-q_{i}\right) t+X_{t}^{(i)}+\omega_{i} t\right)
$$

where $r$ is the constant continuously compounded interest rate, and we assume the asset pays out a continuous dividend yield of $q_{i}$. The risk-neutral drift rate for the asset is $r-q_{i}$ and thus to have $E\left[A_{t}\right]=A_{0} \exp \left(\left(r-q_{i}\right) t\right)$, we have to set

$$
\omega_{i}=\nu^{-1} \log \left(1-\frac{1}{2} \sigma_{i}^{2} \nu-\theta_{i} \nu\right)
$$

\subsection{Dependence Structure}

In this section, we investigate in detail the dependence or association structure caused by the common Gammatime change. We will start from the remark that, conditionally on the time change, the price processes are independent. We will also work, for the sake of simplicity, on their risk neutral log-returns, defined as

$$
Z_{t}^{(i)}:=\log \left(A_{t}^{(i)} / A_{0}^{(i)}\right)
$$

We focus on the $t=1$ situation. Our purpose is that of writing down the (risk neutral) joint distribution function of the vector $\left(Z_{1}^{(1)}, Z_{1}^{(2)}\right), F\left(z_{1}, z_{2}\right)$, possibly using the unconditional marginal distributions, $F^{(i)}\left(z_{i}\right)$, which are easy to obtain.

Conditional normality of log-returns allows us to perform the task in a straightforward way. Let $m_{i}=$ $r-q_{i}+\omega_{i}$. The distribution of each single process, conditional on a realization $x$ of the (Gamma) time change, is Normal, with mean $m_{i}+\theta_{i} x$ and variance $x \sigma_{i}^{2}$. Let us denote it as $F^{(i)}\left(z_{i} \mid x\right)$ :

$$
P_{Q}\left(Z_{1}^{(i)} \leq z_{i} \mid G_{1}=x\right)=F^{(i)}\left(z_{i} \mid x\right)=\Phi\left(\frac{z_{i}-m_{i}-\theta_{i} x}{\sigma_{i} \sqrt{x}}\right)
$$

where $\Phi$ is the cumulative distribution function of the standard Normal random variable.

As for the unconditional distribution, $F^{(i)}\left(z_{i}\right)$, we have

$$
\begin{aligned}
P_{Q}\left(Z_{1}^{(i)} \leq z_{i}\right)=F^{(i)}\left(z_{i}\right) & =\int_{0}^{+\infty} F^{(i)}\left(z_{i} \mid x\right) \frac{\nu^{-1 / \nu}}{\Gamma(1 / \nu)} x^{\frac{1}{\nu}-1} \exp (-x / \nu) \mathrm{d} x \\
& =\int_{0}^{+\infty} \Phi\left(\frac{z_{i}-m_{i}-\theta_{i} x}{\sigma_{i} \sqrt{x}}\right) \frac{\nu^{-1 / \nu}}{\Gamma(1 / \nu)} x^{\frac{1}{\nu}-1} \exp (-x / \nu) \mathrm{d} x .
\end{aligned}
$$

A closed form expression for this integral has been given in Madan, Carr, Chang [6], in terms of Hypergeometric functions and the modified Bessel functions. We note that the integral can also be computed very fast using the inverse Fourier transform approach or via Partial-Differential Integral Equations (PDIEs).

Starting from the marginal conditional distributions (4) also the joint unconditional one can be obtained, since conditional independency holds:

$$
\begin{aligned}
& P_{Q}\left(Z_{1}^{(1)} \leq z_{1}, Z_{1}^{(2)} \leq z_{2}\right)=F\left(z_{1}, z_{2}\right)= \\
& \quad \int_{0}^{+\infty} \prod_{i=1}^{2} \Phi\left(\frac{z_{i}-m_{i}-\theta_{i} x}{\sigma_{i} \sqrt{x}}\right) \frac{\nu^{-1 / \nu}}{\Gamma(1 / \nu)} x^{\frac{1}{\nu}-1} \exp (-x / \nu) \mathrm{d} x .
\end{aligned}
$$

The reader can appreciate the fact that, consistently with the construction method, this distribution depends on the same parameters of the marginal ones: this will be the key to our calibration procedure via marginal probabilities (and marginal derivatives).

As concerns the possibility of writing down the distribution $F\left(z_{1}, z_{2}\right)$ in terms of the marginal ones, $F^{(i)}\left(z_{i}\right)$, this can be accomplished via the copula technique. For a general introduction to copulas, we refer the reader 
to Nelsen [27], for its applications in finance to Cherubini, Luciano, Vecchiato [10]. Let us first notice that the previous expression already contains the conditional marginal distributions, via the factor $\operatorname{copula} C^{\perp}(u, v):=$ $u \times v$ :

$$
\begin{aligned}
& F\left(z_{1}, z_{2}\right)= \\
& \quad \int_{0}^{+\infty} C^{\perp}\left(\Phi\left(\frac{z_{1}-m_{1}-\theta_{1} x}{\sigma_{1} \sqrt{x}}\right), \Phi\left(\frac{z_{2}-m_{2}-\theta_{2} x}{\sigma_{2} \sqrt{x}}\right)\right) \frac{\nu^{-1 / \nu}}{\Gamma(1 / \nu)} x^{\frac{1}{\nu}-1} \exp (-x / \nu) \mathrm{d} x .
\end{aligned}
$$

However, by the properties of the copula functions, in particular Sklar's theorem, we know that there exists a copula, which represents the asset processes' dependence, and we will therefore denote it as $C^{A}$, such that the joint distribution can be written also in terms of the unconditional marginal ones:

$$
F\left(z_{1}, z_{2}\right)=C^{A}\left(F^{(1)}\left(z_{1}\right), F^{(2)}\left(z_{2}\right)\right)
$$

In turn, we know that the copula $C^{A}$ can be obtained as

$$
C^{A}(u, v)=F\left(G^{(1)}(u), G^{(2)}(v)\right),
$$

where the function $G^{(i)}$ is the inverse of $F^{(i)}$. Only this copula represents correctly the dependence between log-returns. Indeed, the linear correlation coefficient between $Z_{1}^{(1)}$ and $Z_{1}^{(2)}$, which can be computed to be

$$
\rho=\frac{\theta_{1} \theta_{2} \nu}{\sqrt{\sigma_{1}^{2}+\theta_{1}^{2} \nu} \sqrt{\sigma_{2}^{2}+\theta_{2}^{2} \nu}}
$$

does not represent it correctly, as one can immediately understand from the fact that it is zero whenever the VG variables are symmetric $\left(\theta_{1}=\theta_{2}=0\right)$. Nonetheless, in the symmetric case, as well as in the non-symmetric one, they are associated, since they are partially driven by the same common factor. Note also that in the typical realistic case of negatively skewed underlying distributions we have a positive correlation.

\subsubsection{Contour Plots}

Contour plots of the copula function give a first, immediately perceivable information on the copula behavior, since we know that:

- the contours of the minimum copula, $C^{-}(u, v):=\max (u+v-1,0)$, which represents maximal negative association or countermonotonicity, are the straight lines with equation $u=c-1-v$, for $c \in[0,1]$;

- those of the maximum copula, $C^{+}(u, v):=\min (u, v)$, which represents maximal positive association or comonotonicity, are kinked lines, which form a $90^{\circ}$ angle on the main diagonal of the unit square;

- those of any other copula are included between the previous ones. The the closer they are to the former (latter) ones the more negatively (positively) associated are the corresponding random variables.

To investigate the effect of the different parameters on the copula (7), therefore, we numerically evaluate the copula function and its contour lines for various values of the parameters $\sigma_{i}, \theta_{i}, \nu$, as follows:

- for a grid $\left\{\left(u_{i}, v_{j}\right), i, j=1, \ldots, N\right\}$ on $[0,1] \times[0,1]$ we calculate (numerically) the inverse functions $G^{(1)}\left(u_{i}\right)$ and $G^{(2)}\left(v_{i}\right)$

- we compute $C_{i j}=F\left(G^{(1)}\left(u_{i}\right), G^{(2)}\left(v_{j}\right)\right)$;

- we contour plot $C_{i j}$ versus the grid.

The results of this procedure can be seen in Figure 2. In each figure, we plotted several level curves, on which the couples $\left(u_{i}, v_{j}\right)$ give rise to a predetermined constant copula value. 


\subsubsection{Scatter Plots}

Scatter plots, which are plots of the realizations $(u, v)$ for a specific copula, are informative of the dependence behavior too: essentially, the more dispersion one observes, less dependence can be inferred. The more points one observes on increasing (decreasing) lines - or close to them - the more positively (negatively) associated are the underlying random variables. The more the points cluster in the corners of the unit square, the more evident tail dependency is.

Scatter plots of the copula ( 7 ) can be constructed, for fixed values of the parameters $\sigma_{i}, \theta_{i}, \nu$, as follows:

- simulate $M$ couples of VG realizations from processes of the type (1). Let them be $\left(x_{m}^{(1)}, x_{m}^{(2)}\right), m=$ $1, \ldots, M$

- compute their inverses according to the $F^{(i)}$ functions:

$$
u_{m}^{(i)}:=F^{(i)}\left(x_{m}^{(i)}\right)
$$

These are the cumulative probabilities associated to the realizations $x_{m}^{(i)}$. It follows that the couples $\left(u_{m}^{(1)}, u_{m}^{(2)}\right)$ are random realizations from the copula $C^{A}$.

The results of this procedure, with $M=1000$ can be seen in Figure 3

\subsubsection{Concordance and Tail Dependence Measures}

Copulas can be used to define concordance measures, such as Spearman's rho and Kendall's tau. These two measures are bounded between -1 and 1, with -1 representing countermonotonicity and 1 representing comonotonicity: the correspondence is one-to-one, provided that the underlying random variables, as in our case, are continuous. It follows that concordance measures can make the information provided by contour and scatter plots precise. Let us focus on Spearman's rho, the rank correlation coefficient, recalling that it is defined as follows, in copula terms:

$$
\rho_{S}=12 \iint_{I^{2}} C(v, z) \mathrm{d} v \mathrm{~d} z-3=12 \iint_{I^{2}} v z \mathrm{~d} C(v, z)-3 .
$$

Table 1 below reproduces the empirical versions of the Spearman's coefficient, using (9), for the cases of Figures 2 and 3, together with the corresponding linear correlation coefficient, computed according to formula (8).

\begin{tabular}{llllllll}
\hline \hline$\sigma_{1}$ & $\theta_{1}$ & $\sigma_{2}$ & $\theta_{2}$ & $\nu$ & $\rho$ & $\rho_{S}$ & $\lambda_{L}$ \\
\hline 0.2 & -0.10 & 0.25 & -0.15 & 1 & 0.23 & 0.04 & 0.16 \\
0.2 & -0.10 & 0.25 & -0.15 & 2.5 & 0.43 & 0.10 & 0.21 \\
0.2 & -0.25 & 0.25 & -0.20 & 1 & 0.49 & 0.14 & 0.20 \\
0.2 & -0.25 & 0.25 & -0.20 & 2.5 & 0.70 & 0.25 & 0.38 \\
0.2 & -0.35 & 0.25 & -0.30 & 1 & 0.67 & 0.45 & 0.32 \\
0.2 & -0.35 & 0.25 & -0.30 & 2.5 & 0.83 & 0.49 & 0.55 \\
\hline \hline
\end{tabular}

Table 1: Correlation, Spearman's rho and lower tail depencence

To end up with, copulas can be used to define upper and lower tail dependency, which intuitively correspond to dependence of extreme events, as follows. $C$ is said to have upper tail dependence if and only if

$$
\lambda_{U}:=\lim _{v \rightarrow 1-} \frac{C(v, v)+1-2 v}{1-v}
$$

exists positive, and lower tail dependence if and only if

$$
\lambda_{L}:=\lim _{v \rightarrow 0+} \frac{C(v, v)}{v}
$$


exists positive. The latter dependence coefficient is particularly interesting for prices and returns, since it corresponds to concordance in market crashes. Its empirical version can therefore formalize the existence, in the multivariate VG model built so far, of crash dependence. For the VG cases of Figures 2 and 3 the values of $\lambda_{L}$, which were obtained by numerically evaluating the limit in (10), are also collected in Table 1.

\subsubsection{Sensitivity Analysis of the Copula}

From Figures 2 and 3, as well as from Table 1, we can attain some feeling on the behavior of the copula (7).

Sensitivity w.r.t. $\nu$ : The behavior of returns with respect to the level of economic activity, i.e. the common Gamma transform, is mainly measured in terms of $\nu$. An increase in $\nu$ increases marginal variance and kurtosis, according to the expressions in Section 2.2 above. It also increases concordance: the effect is evident on both the level curves of Figure 2 and on the corresponding copula realizations of Figure 3. Indeed, when passing from the left column, which corresponds to $\nu=1$, to the right one, which corresponds to $\nu=2.5$, the copula level curves of Figure 2 become more kinked, and therefore closer to the maximum copula ones: this could be confirmed by plotting on the same graph the level curves of the maximum copula, or by calculating mean square errors. Also, from the first to the second column, the points of the scatter plots in Figure 3 tend to cluster along the main diagonal, and in particular - along it - towards the origin and at a second point, close to the upper corner of the square. This - probably bimodal - behavior explains the appearance in the second column graphs of a sort of "anchor", more pronounced for higher values of the asymmetry parameters $\theta_{i}$.

The increase in concordance is well captured by the empirical version of Spearman's rho, which goes, according to Table 1 , from $4 \%$ to $10 \%$ in the first row case (low $\theta_{i}$ 's), from $14 \%$ to $25 \%$ in the second line one (medium $\theta_{i}$ 's), from $45 \%$ to $49 \%$ in the last line one (high $\theta_{i}$ 's). It is also confirmed by the linear correlation correlation coefficient, as the reader can check in Table 1.

Last but not least, increasing $\nu$ seems to increase also the lower tail dependence, since the scatter plot points cluster in the lower left corner when going from the left to the right column, starting from an already more clustered situation in the lower rows, i.e. when the asymmetry parameter is higher. This is confirmed by the tail dependence estimators of Table 1.

Sensitivity w.r.t. $\theta_{i}$ : When we increase in absolute value the (negative) asymmetry coefficients $\theta_{i}$, i.e. when we go from top to bottom in each column of Figures 2 and 3, we observe again increasing concordance, since level curves become more kinked and scatter points cluster on the main diagonal. This is consistent with intuition, since increasing $\theta_{i}$ in $X^{(i)}=\theta_{i} G_{t}+\sigma_{i} W_{G_{t}}^{(i)}$ decreases - ceteris paribus - the role of the idiosyncratic component.

In Figure 3, we can also remark an increase in lower tail dependency, since the points tend to cluster around the origin. Both increases, in concordance and lower tail dependence, are confirmed by the coefficients of Table 1 .

As said above, the presence of lower tail dependency is a particularly nice feature of the multivariate model just built, which makes it particularly prone to use in equity and credit modelling, in order to incorporate higher dependence of respectively low returns and defaults than of high returns and non-default situations.

The sensitivity w.r.t. $\sigma_{i}$ is not explored via the above cases, but can be obtained from the Authors upon request: as expected, the copula does not seem to be very sensitive to changes in this parameter, which drives the idiosyncratic Brownian factors, $W^{(1)}$ and $W^{(2)}$, but leaves unchanged the common Gamma one, $G$.

Remark 1 We note here that it is tempting to compare the copula (7) with the Archimedean Clayton one, which arises when mixing Exponentials random variates by a Gamma distribution (see [29, Theorem 10.5] and [25]). As wrongly reported in [15] other mixtures, like a mixture of Normals with a Gamma, as in our setting, do not lead to a Clayton copula.

\section{Applications}

\subsection{Equity Modelling}

The model proposed above can be used in order to describe the joint behavior of equities or equity indexes. It will be possible to calibrate it directly under the risk neutral measure and using data on univariate derivative 
products due to the fact that, as stressed above, its marginal and joint distributions at any fixed point in time depend on the same parameters. This will allow us to price rainbow products, such as options on the maximum, on the minimum, options on the best or worst of, without introducing the adhockery of no premium for market crashes and default dependence.

\subsubsection{The Multivariate VG-Model}

We model $n$ dependent stock price, $\left(S_{t}^{(1)}, \ldots, S_{t}^{(n)}\right)$ process as in $(2)$. More precisely, we have

$$
S_{t}^{(i)}=S_{0} \exp \left(\left(r-q_{i}\right) t+\theta_{i} G_{t}+\sigma_{i} W_{G_{t}}^{(i)}+\omega_{i} t\right)
$$

where $\left(W_{t}^{(1)}, \ldots, W_{t}^{(n)}\right)$ is a vector of independent Brownian Motions and $G=\left\{G_{t}, t \geq 0\right\}$ is a common Gamma process with parameters $a=b=1 / \nu$ independent from all Brownian motions involved ${ }^{1}$.

The multivariate risk neutral distribution of such stock prices, having defined log returns as in (3), is a straightforward extension of (5).

The pricing of European-type Basket-options can easily be done by Monte-Carlo simulation. To simulate the paths of $n$ dependent stock prices according to the above model, one can proceed as follows.

Discretize the time-interval $[0, T]$ in to $N$ equally spaced intervals: Let $\Delta t=T / N$ and set $t_{j}=j \Delta t$, $j=0, \ldots, N$. Set $S_{i, 0}=S_{0}^{(i)}$. Calculate $S_{i, j}=S_{t_{j}}^{(i)}$ : for $j$ from 1 to $N$ do the following:

- sample a random number $g_{j}$ out of the $\operatorname{Gamma}(\Delta t / \nu, 1 / \nu)$ distribution;

- sample for each $i=1, \ldots, n$ an independent standard Normal random number $v_{i, j}$.

- Set

$$
S_{i, j}=S_{i, j-1} \exp \left(\left(r-q_{i}\right) \Delta t+\theta_{i} g_{j}+\sigma_{i} \sqrt{g_{j}} v_{i, j}+\omega_{i} \Delta t\right)
$$
1 .

This will give a realization of the dependent stock prices at the chosen time-grid, as can be seen in Figure

\subsubsection{The Stochastic Volatility VG-Model}

One can even go a step further and build in stochastic volatility in the above model. Incorporating stochastic volatility effects into the price process can be done by making one more stochastic time change. Periods with high volatility can be interpreted as if time runs faster than in periods with low volatility. Applications of stochastic time change to asset pricing go back to Mandelbrot and Taylor [24] (see also Clark [11]). We consider the model introduced by Carr, Geman, Madan and Yor [5].

In order to have stochastic volatility we will have, on top of the setting in the previous sections, for each individual price, an independent time-change taking care of the companies own stochastic volatility ${ }^{2}$. The behavior of the price processes will then be modeled by the exponential of dependent Lévy process suitably (and independently) time-changed. Note that the time-change needed to have stochastic volatility effects is of an other nature than the one in Section 2; here we need a mean-reverting effect.

Following [5], we opt here to use for the rate of time change of the $i$ th price a CIR-process $y^{(i)}=\left\{y_{t}^{(i)}, t \geq 0\right\}$ that solves the SDE:

$$
\mathrm{d} y_{t}^{(i)}=\kappa_{i}\left(\eta_{i}-y_{t}^{(i)}\right) \mathrm{d} t+\lambda_{i}\left(y_{t}^{(i)}\right)^{1 / 2} \mathrm{~d} \tilde{W}_{t}^{(i)}
$$

where $\tilde{W}^{(i)}=\left\{\tilde{W}_{t}^{(i)}, t \geq 0\right\}$ are independent standard Brownian motions independent of all other process which are into play. The economic time corresponding to calendar time $t$ is then

$$
Y_{t}^{(i)}:=\int_{0}^{t} y_{s}^{(i)} \mathrm{d} s .
$$

\footnotetext{
${ }^{1}$ Note that in theory we can make the Brownian motions dependent on each other (as in [22]). However, this would lead to a quadratic increase in the parameters and would generate an estimation problem of the correlation structure, as discussed before.

${ }^{2}$ Extensions to common stochastic volatility time changes can be part of future research.
} 
The characteristic function of $Y_{t}^{(i)}$ (given $y_{0}^{(i)}$ ) is explicitly known (see [12]):

$$
\begin{aligned}
\varphi_{C I R}\left(u, t ; \kappa_{i}, \eta_{i}, \lambda_{i}, y_{0}^{(i)}\right) & =E\left[\exp \left(\mathrm{i} u Y_{t}^{(i)}\right) \mid y_{0}^{(i)}\right] \\
& =\frac{\exp \left(\kappa_{i}^{2} \eta_{i} t / \lambda_{i}^{2}\right) \exp \left(2 y_{0}^{(i)} \mathrm{i} u /\left(\kappa_{i}+\gamma_{i} \operatorname{coth}\left(\gamma_{i} t / 2\right)\right)\right)}{\left(\cosh \left(\gamma_{i} t / 2\right)+\kappa_{i} \sinh \left(\gamma_{i} t / 2\right) / \gamma_{i}\right)^{2 \kappa_{i} \eta_{i} / \lambda_{i}^{2}}}
\end{aligned}
$$

where

$$
\gamma_{i}=\sqrt{\kappa_{i}^{2}-2 \lambda_{i}^{2} \mathrm{i} u}
$$

Due to (time)-scaling effects, one can w.l.o.g. scale the present rate of time change to 1 , i.e. set $y_{0}^{(i)}=1$. Other possible time-changes, based on Ornstein Uhlenbeck-type processes (which can be jump process) as in [30], can readily be used to.

The (risk-neutral) price process $S^{(i)}=\left\{S_{t}^{(i)}, t \geq 0\right\}$ is now modeled as follows:

$$
S_{t}^{(i)}=S_{0}^{(i)} \frac{\exp \left(\left(r-q_{i}\right) t\right)}{E\left[\exp \left(X_{Y_{t}^{(i)}}^{(i)}\right) \mid y_{0}^{(i)}\right]} \exp \left(X_{Y_{t}^{(i)}}^{(i)}\right)
$$

where $X^{(i)}=\left\{X_{t}^{(i)}=\theta_{i} G_{t}+\sigma_{i} W_{G_{t}}^{(i)}, t \geq 0\right\}$ is a VG process. The factor $\exp \left(\left(r-q_{i}\right) t\right) / E\left[\exp \left(X_{Y_{t}^{(i)}}^{(i)}\right) \mid y_{0}^{(i)}\right]$ puts us immediately into the risk-neutral world by a mean-correcting argument. Essentially, the price process is modeled as the ordinary exponential of a time-changed Lévy process (a VG process). The process incorporates jumps (through the Lévy process) and stochastic volatility (through the time change).

The corresponding log returns are

$$
Z_{t}^{(i)}:=\log E\left[\exp \left(X_{Y_{t}^{(i)}}^{(i)}\right) \mid y_{0}^{(i)}\right]+\left(r-q_{i}\right) t+X_{Y_{t}^{(i)}}^{(i)}
$$

The characteristic function $\phi(u, t)$ of the log-price process is given by:

$$
\begin{aligned}
\phi(u, t) & =E\left[\exp \left(\mathrm{i} u \log \left(S_{t}^{(i)}\right)\right) \mid S_{0}^{(i)}, y_{0}^{(i)}\right] \\
& =\exp \left(\mathrm{i} u\left(\left(r-q_{i}\right) t+\log S_{0}^{(i)}\right)\right) \frac{\varphi^{(i)}\left(-\mathrm{i} \psi_{X}^{(i)}(u) ; t, y_{0}^{(i)}\right)}{\varphi^{(i)}\left(-\mathrm{i} \psi_{X}^{(i)}(-\mathrm{i}) ; t, y_{0}^{(i)}\right) \mathrm{i} u}
\end{aligned}
$$

where

$$
\psi_{X}^{(i)}(u)=\log E\left[\exp \left(\mathrm{i} u X_{1}^{(i)}\right)\right]
$$

and $\varphi^{(i)}\left(u ; t, y_{0}^{(i)}\right)$ denotes the characteristic function of $Y_{t}^{(i)}$ given $y_{0}^{(i)}$ (for more details, see [5] or [30]).

The log-return distribution at time 1 could be constructed by conditioning first on the integrated CIR process, analogously to what we did for the VG process. By doing so, at the multivariate level, one could exploit once more conditional independence in order to arrive at a representation of the type (5).

\subsection{Credit Risk Modelling}

There are basically two main classes of models for credit pricing: intensity based models and firm value models. Here, we essentially apply the model presented above to produce a new dynamic multivariate firm value model in the style of Merton, where default is triggered by the fact that the firm value at maturity is too low, and in the style of Black and Cox, in which default is triggered by the hitting of a low barrier.

Most available firm value models for credit pricing have a stochastic process with continuous paths modelling the firm's asset value. However, one often would like to have a more flexible underlying distribution than the Normal distribution in order to take into account skewness and excess kurtosis. Moreover, default events are most of the time triggered by shocks and hence one would like to have also jumps in the asset value. This can be accomplished by adding a Poisson component, as in Zhou [31]. By doing so, one does not overcome another 
modelling problem: very often, the dependence structure of the assets is used in order to infer the one of the default intensities (see for instance Li [17]). However, this is not correct, since, as pointed out by Duffie and Lando [14], a continuous path asset value does not produce a totally inaccessible default time, and therefore does not admit a corresponding intensity. Neither a continuous asset process with superimposed jumps would have an intensity. Only a pure jump structural model would admit an intensity.

We build a new multivariate Merton-type model and Black-Cox-type model, where we assume that the asset price processes are described by an exponential of a dependent (non-Brownian) Lévy processes. As such, we take into account asymmetry and fat-tail behaviour. An other advantage by following this approach is the fact that in contrast to Brownian motion the underlying Lévy process is a jump processes and hence instantaneous default is possible. Dependence is build by making use of the common time-change. Again, we work out the details in the popular case of a Variance Gamma Lévy process.

Default can be triggered either by the fact that the firm value at maturity is too low to cover the facial value of debt, as in the traditional Merton's model, or by the hitting of a deterministic low-barrier, as in the traditional Black-Cox's model. In the second case early default is possible. After briefly introducing the Mertontype model, in what follows we will put most emphasis on the more involved Black-Cox-type model. Under this model we will discuss the pricing of a Credit Default Swap (CDS). The pricing is based on the solution of a PDIE. CDSs are the basic derivatives in the credit market on which one typically likes to calibrate the model on. We stress here that in under our model we are able of calibrating on the CDS derivatives available in the market only; we do not need historical data.

Next, we start with a brief description of a Merton's type of which the univariate case was introduced, for the VG process, in Madan [19]. We will model in this context $n$ firms; the risk-neutral firm value of the processes are modelled by (2).

\subsubsection{Multivariate Merton-type VG Firm Value Model}

Suppose the $i$ th firm has a unique, zero-coupon debt issue with facial value $L_{i}$ with maturity $T$. Then, debt holders receive either $L_{i}$ or the asset value $A_{T}^{(i)}$, if the latter is smaller than the former. Therefore, the time to default of the $i$ th firm, $\tau_{i}$ is given by

$$
\tau_{i}=\left\{\begin{array}{cc}
T & A_{T}^{(i)}<L_{i} \\
+\infty & A_{T}^{(i)} \geq L_{i} .
\end{array}\right.
$$

Debt holders on the $i$ th firm in turn have a claim of $L_{i}$ and are short a put on the firm value, with final payoff

$$
\max \left(L_{i}-A_{T}^{(i)}, 0\right)
$$

The debt value at any time before maturity coincides with this put price, while the default probability is the corresponding exercise probability. Closed-form formulas for both have been provided by Madan, Carr and Chang [6].

Under this model, the marginal conditional, $p_{i}^{T}(x)$, (on $G_{T}=x$ ), the marginal unconditional, $P_{i}^{d}(T)$, and the joint unconditional, $P^{d}(T)$, risk neutral default probabilities are resp. given by:

$$
\begin{aligned}
p_{i}^{T}(x) & =F^{(i)}\left(L_{i} \mid x\right)=\Phi\left(\frac{l_{i}-\left(r-q_{i}+\omega_{i}\right) T-\theta_{i} x}{\sigma_{i} \sqrt{x}}\right) \\
P_{i}^{d}(T) & =F^{(i)}\left(L_{i}\right)=\int_{0}^{+\infty} \Phi\left(\frac{l_{i}-\left(r-q_{i}+\omega_{i}\right) T-\theta_{i} x}{\sigma_{i} \sqrt{x}}\right) \frac{\nu^{-T / \nu}}{\Gamma(T / \nu)} x^{\frac{T}{\nu}-1} \exp (-x / \nu) \mathrm{d} x \\
P^{d}(T) & =\int_{0}^{+\infty} \prod_{i=1}^{n} \Phi\left(\frac{l_{i}-\left(r-q_{i}+\omega_{i}\right) T-\theta_{i} x}{\sigma_{i} \sqrt{x}}\right) \frac{\nu^{-T / \nu}}{\Gamma(T / \nu)} x^{\frac{T}{\nu}-1} \exp (-x / \nu) \mathrm{d} x
\end{aligned}
$$

where $l_{i}=\ln \left(L_{i} / A_{0}^{(i)}\right)$. 


\subsubsection{Multivariate VG Barrier Model}

Let us now move to the barrier model, where the barrier corresponds to the recovery value of the firm's debt, net of bankruptcy costs. More precisely, the $i$ th firm defaults the first time its asset value process $A^{(i)}=\left\{A_{t}^{(i)}, t \geq 0\right\}$ hits a low barrier $H_{i}<A_{0}^{(i)}$. The time of default of the $i$ th firm, $\tau_{i}$, is hence given by

$$
\tau_{i}=\inf \left\{t \geq 0 \mid A_{t}^{(i)} \leq H_{i}\right\} .
$$

Let us denote by $P_{i}^{s}(t)$ the risk-neutral survival probability between 0 and $t$ :

$$
\begin{aligned}
P_{i}^{s}(t) & =P_{Q}\left(A_{s}^{(i)}>H_{i}, \text { for all } 0 \leq s \leq t\right) ; \\
& =E_{Q}\left[\mathbf{1}\left(\min _{0 \leq s \leq t} A_{s}^{(i)}>H_{i}\right)\right]
\end{aligned}
$$

where we used again the indicator function $\mathbf{1}(B)$; the subindex $Q$ refers to the fact that we are working in a risk-neutral setting.

Differently from the Merton-type case, the probability $P^{s}(t)$ cannot be written in closed form: however, it can be easily computed, either via Montecarlo techniques or as the solution of an appropriate PIDE (see [7]). The second approach saves computational time. The probability $P_{i}^{s}(t)$ will be the main ingredient in the pricing formula of the CDSs on which we will calibrate our models.

\section{Calibration}

Next, we will calibrate the models to a set of basic derivatives: vanilla options in Equity and CDS term structures in Credit. We use the Nelder-Mead simplex (direct search) method to minimize the difference between market prices and model prices in a least-squares sense: we minimizing the root mean square error ( $r m s e)$ given by

$$
\text { rmse }=\sqrt{\sum_{\text {derivatives }} \frac{(\text { Market price }- \text { Model price })^{2}}{\text { number of derivatives }}} .
$$

\subsection{Equity: Calibration on Vanilla Options}

Carr and Madan [6] developed pricing methods for the classical vanilla options which can be applied in general when the characteristic function of the risk-neutral stock price process is known.

Let $\alpha$ be a positive constant such that the $\alpha$ th moment of the stock price exists. Carr and Madan then showed that the price $C(K, T)$ of a European call option with strike $K$ and time to maturity $T$ is given by:

$$
C(K, T)=\frac{\exp (-\alpha \log (K))}{\pi} \int_{0}^{+\infty} \exp (-\mathrm{i} v \log (K)) \varrho(v) \mathrm{d} v,
$$

where

$$
\begin{aligned}
\varrho(v) & =\frac{\exp (-r T) E\left[\exp \left(\mathrm{i}(v-(\alpha+1) \mathrm{i}) \log \left(S_{T}\right)\right)\right]}{\alpha^{2}+\alpha-v^{2}+\mathrm{i}(2 \alpha+1) v} \\
& =\frac{\exp (-r T) \phi(v-(\alpha+1) \mathrm{i}, T)}{\alpha^{2}+\alpha-v^{2}+\mathrm{i}(2 \alpha+1) v} .
\end{aligned}
$$

Using Fast Fourier Transforms, one can compute within a second the complete option surface on an ordinary computer. We apply the above calculation method in our calibration procedure. The only difference with a univariate calibration is that we simultaneously calibrate all stocks by using a common $\nu$-parameter

\subsubsection{The Multivariate VG Model}

In a univariate setting the VG model is know for its ability to fit option prices (or equivalently implied volatilities) at one maturity. In order to illustrate that our multivariate model is also able of doing that, we fit the model on a data set of vanilla options on the SP-500, the Nikkei-225 and the Eurostoxx-50, all taken at the same instant (April, 5, 2005). The maturity of the involved options are approximately one year. As can be seen from Figure 4 , our model is capable of simultaneously fitting all market options at the given maturity very well. 


\subsubsection{The Multivariate Stochastic Volatility VG Model}

The VG model is not really able of globally fitting all option prices over all maturities. Allthough the fits are much better than in the classical Black-Scholes setting (see e.g. [30]), the fact that no stochastic volatility is build in still leads in a global calibration to some discrepancy between model and market prices, especially at long maturities. This was one of the driving motivations to include stochastic volatility (by time-changing) into the VG model. As in the univariate case our multivariate VG model with stochastic volatility (VG-SV) can nicely fit the market situation and this over all maturities and all strikes. Again we fit the model on a data set of vanilla options on the SP-500, the Nikkei-225 and the Eurostoxx-50, again taken at the same instant (April, 5,2005 ), but now taking into account maturities ranging from one month to over five years. As can be seen from Figure 5, our model is capable of simultaneously fitting all market options over all maturities very well.

\begin{tabular}{lrrrrrrr}
\hline \hline Company & $\sigma$ & $\nu$ & $\theta$ & $\kappa$ & $\eta$ & $\lambda$ \\
\hline Eurostoxx-50 & 0.1306 & 0.1665 & -0.1287 & 1.0701 & 0.8437 & 1.9926 & 1 \\
Nikkei-225 & 0.1642 & 0.1665 & -0.0429 & 0.3395 & 1.2268 & 1.0616 & 1 \\
SP-500 & 0.1421 & 0.1665 & -0.0816 & 0.9802 & 0.8667 & 2.4049 & 1 \\
\hline \hline
\end{tabular}

Table 2: Calibration on Eurostoxx50, Nikkei 225 and SP-500

The results obtained for the process parameters, which can be found in Table 2 are in line with previous implementations of both the VG and its stochastic volatility version. The value of $\nu$ obtained for the $\mathrm{VG}$ case is $\nu=0.7514$, while in the stochatsic volatility case we get $\nu=0.1665$. In the former case we know from the dependence analysis of section 2.4.4 that that value of the $\nu$ parameter implies quite a strong dependence, which could be further explored using the simulation tools of section 2.4: we can tentatively infer also a weaker concordance with stochastic volatility.

\subsection{Credit: Calibration on CDS Term Structure Curve}

In the Credit setting, the CDSs will be the basic derivatives on which we will calibrate our model. Consider a CDS with maturity $T$ and a continuous spread $c$. The price of a this CDS is then given by

$$
C D S=(1-R)\left(-\int_{0}^{T} \exp (-r s) \mathrm{d} P(s)\right)-c \int_{0}^{T} \exp (-r s) P(s) \mathrm{d} s,
$$

where $R$ is the asset specific recovery rate, $P(s)$, the firm's survival probability and $r$ is the default-free discount rate.

From this, we find the par spread $c^{*}$ that makes the CDS price equal to zero:

$$
\begin{aligned}
c^{*} & =\frac{(1-R)\left(-\int_{0}^{T} \exp (-r s) \mathrm{d} P(s)\right)}{\int_{0}^{T} \exp (-r s) P(s) \mathrm{d} s} \\
& =\frac{(1-R)\left(1-\exp (-r T) P(T)-r \int_{0}^{T} \exp (-r s) P(s) \mathrm{d} s\right)}{\int_{0}^{T} \exp (-r s) P(s) \mathrm{d} s}
\end{aligned}
$$

In [7] a fast numerical method for the pricing of CDSs under a VG model was described. The technique is based on the pricing of digital barrier options by solving Partial Integro-Differential Equations (PIDEs).

The advantage here is that we can still use this technique for the pricing of individual CDSs. Moreover, calibration can easily be done by the same pricing technique in a multivariate setting. One just has to calibrate all the CDS curves simultaneous using a common $\nu$-parameter.

Next, we will calibrate the model to a basket of CDS term structures which were taken from the market situation on the 26th of October 2004. In Figure 6, the joint calibration for a set of five companies is visualized. In Table 4, one finds the parameters coming out of the calibration procedure; Table 3 reports the corresponding market and models prices. One sees clearly, that the model's CDS term structure curve captures nicely the market quotes (o-signs). 


\begin{tabular}{lllrrrrr}
\hline \hline Company & Moody & & $1 y$ & $3 y$ & $5 y$ & $7 y$ & $10 y$ \\
\hline Autozone & Baa2 & Market & 25 & 65 & 102 & 117 & 127 \\
Autozone & Baa2 & Model & 21 & 69 & 101 & 117 & 126 \\
\hline Ford Credit Co. & A3 & Market & 75 & 154 & 203 & 225 & 238 \\
Ford Credit Co. & A3 & Model & 66 & 165 & 209 & 224 & 224 \\
\hline Kraft & A3 & Market & 4 & 19 & 31 & 40 & 51 \\
Kraft & A3 & Model & 4 & 18 & 32 & 41 & 50 \\
\hline Walt Disney & Baa2 & Market & 6 & 21 & 36 & 45 & 56 \\
Walt Disney & Baa2 & Model & 5 & 21 & 36 & 46 & 55 \\
\hline Whirlpool & Baa1 & Market & 16 & 36 & 66 & 73 & 86 \\
Whirlpool & Baa1 & Model & 12 & 40 & 63 & 76 & 85 \\
\hline \hline
\end{tabular}

Table 3: Calibration on CDS (in bp) term structure

\begin{tabular}{lrrr}
\hline \hline Company & $\sigma$ & $\nu$ & $\theta$ \\
\hline AutoZone & 0.2025 & 0.7068 & -0.0250 \\
Ford & 0.2562 & 0.7068 & -0.0252 \\
Kraft & 0.1510 & 0.7068 & -0.0296 \\
Walt Disney & 0.1543 & 0.7068 & -0.0330 \\
Whirlpool & 0.1745 & 0.7068 & -0.0396 \\
\hline \hline
\end{tabular}

Table 4: Calibration on CDS (in bp) term structure

\section{Conclusions and Further Research}

We have presented a multivariate Lévy process for modelling financial assets: prices are driven by a common, stochastic time change, which has to be understood as the effect of the economic activity, and which causes sudden jumps in asset values, as well as by idiosyncratic Brownian motions. The model has been fully specified for the case of a gamma time change, thus leading to the multivariate version of the quite popular VG model, both with and without stochastic volatility.

Section 2 has highlighted the theoretical properties of the model, both in terms of analytical tractability, clarity of the dependence structure and parsimony of parameters. Section 3 has pointed its easiness of simulation and speed of calibration.

Based on the calibrations presented above, the model seems to match very well price data, both on the equity and credit derivatives market. Obviously, the empirical analysis has to be pursued further, both extending the database and the goodness of fit analysis: in this sense, we should first of all consider not only (percentage) pricing errors, but also their standard errors.

As concerns the equity risk application, we expect the model to outperform traditional jointly Gaussian structures, mainly because of the inclusions of fat tails, asymmetry and tail dependence.

As concerns the credit risk application, we aim at comparing the model proposed here with some structural, diffusion based ones, in order to check whether current shortcomings of the latter can be overcome by the former. We expect for sure the problem of too low short term credit spreads to be eliminated by the multivariate Lévy proposal.

In both cases, the possibility of calibrating the multivariate model directly under the risk-neutral measure, without having to stick to multivariate normality and without assuming no risk premium for equity or default dependence, will allow us to price multivariate derivatives and credit derivatives in a satisfactory, credible way. 


\section{Appendix}

\section{Lévy Processes}

\section{Definition}

Suppose $\phi(z)$ is the characteristic function of a distribution. If for every positive integer $n, \phi(z)$ is also the $n$th power of a characteristic function, we say that the distribution is infinitely divisible. One can define for every such an infinitely divisible distribution a stochastic process, $X=\left\{X_{t}, t \geq 0\right\}$, called Lévy process, which starts at zero, has independent and stationary increments and such that the distribution of an increment over $[s, s+t]$, $s, t \geq 0$, i.e. $X_{t+s}-X_{s}$, has $(\phi(z))^{t}$ as characteristic function.

The function $\psi(z)=\log \phi(z)$ is called the characteristic exponent and it satisfies the following LévyKhintchine formula [1]:

$$
\psi(z)=\mathrm{i} \gamma z-\frac{\varsigma^{2}}{2} z^{2}+\int_{-\infty}^{+\infty}\left(\exp (\mathrm{i} z x)-1-\mathrm{i} z x 1_{\{|x|<1\}}\right) \nu(\mathrm{d} x)
$$

where $\gamma \in \mathbf{R}, \varsigma^{2} \geq 0$ and $\nu$ is a measure on $\mathbf{R} \backslash\{\mathbf{0}\}$ with $\int_{-\infty}^{+\infty}\left(1 \wedge x^{2}\right) \nu(\mathrm{d} x)<\infty$. We say that our infinitely divisible distribution has a triplet of Lévy characteristics $\left[\gamma, \varsigma^{2}, \nu(\mathrm{d} x)\right]$. The measure $\nu(\mathrm{d} x)$ is called the Lévy measure of $X$. From the Lévy-Khintchine formula, one sees that, in general, a Lévy process consists of three independent parts: a linear deterministic part, a Brownian part, and a pure jump part. The Lévy measure $\nu(\mathrm{d} x)$ dictates how the jumps occur. Jumps of sizes in the set $A$ occur according to a Poisson process with parameter $\int_{A} \nu(\mathrm{d} x)$. If $\varsigma^{2}=0$ and $\int_{-1}^{+1}|x| \nu(\mathrm{d} x)<\infty$ it follows from standard Lévy process theory [1] [28], that the process is of finite variation.

\section{The Variance Gamma Process}

The characteristic function of the $\operatorname{VG}(\sigma, \nu, \theta)$ law is given by

$$
\phi_{V G}(u ; \sigma, \nu, \theta)=\left(1-\mathrm{i} u \theta \nu+\sigma^{2} \nu u^{2} / 2\right)^{-1 / \nu} .
$$

This distribution is infinitely divisible and one can define the VG-process $X^{(V G)}=\left\{X_{t}^{(V G)}, t \geq 0\right\}$ as the process which starts at zero, has independent and stationary increments and where the increment $X_{s+t}^{(V G)}-X_{s}^{(V G)}$ over the time interval $[s, t+s]$ follows a $\operatorname{VG}(\sigma, \nu / t, t \theta)$ law:

$$
\begin{aligned}
E\left[\exp \left(\mathrm{i} u X_{t}^{(V G)}\right)\right] & =\phi_{V G}(u ; \sigma \sqrt{t}, \nu / t, t \theta) \\
& =\left(\phi_{V G}(u ; \sigma, \nu, \theta)\right)^{t} \\
& =\left(1-\mathrm{i} u \theta \nu+\sigma^{2} \nu u^{2} / 2\right)^{-t / \nu} .
\end{aligned}
$$

In [20], it was shown that the VG-process may also be expressed as the difference of two independent Gamma processes. This characterization allows the Lévy measure to be determined:

$$
\nu_{V G}(\mathrm{~d} x)=\left\{\begin{array}{ll}
C \exp (G x)|x|^{-1} \mathrm{~d} x & x<0 \\
C \exp (-M x) x^{-1} \mathrm{~d} x & x>0
\end{array},\right.
$$

where

$$
\begin{aligned}
C & =1 / \nu>0 \\
G & =\left(\sqrt{\frac{\theta^{2} \nu^{2}}{4}+\frac{\sigma^{2} \nu}{2}}-\frac{\theta \nu}{2}\right)^{-1}>0 \\
M & =\left(\sqrt{\frac{\theta^{2} \nu^{2}}{4}+\frac{\sigma^{2} \nu}{2}}+\frac{\theta \nu}{2}\right)^{-1}>0 .
\end{aligned}
$$


The Lévy measure has infinite mass, and hence a VG-process has infinitely many jumps in any finite time interval. Since $\int_{-\infty}^{+\infty}|x| \nu_{V G}(\mathrm{~d} x)<\infty$, a VG-process has paths of finite variation. A VG-process has no Brownian component and its Lévy triplet is given by $\left[\gamma, 0, \nu_{V G}(\mathrm{~d} x)\right]$, where

$$
\gamma=\frac{-C(G(\exp (-M)-1)-M(\exp (-G)-1))}{M G} .
$$

With the parameterization in terms of $C, G$ and $M$, the characteristic function of $X_{1}^{(V G)}$ reads as follows:

$$
\phi_{V G}(u ; C, G, M)=\left(\frac{G M}{G M+(M-G) \mathrm{i} u+u^{2}}\right)^{C} .
$$

In this notation we will refer to the distribution by the notation $\operatorname{VG}(C, G, M)$.

When $\theta=0$ then $G=M$ and the distribution is symmetric. Negative values of $\theta$ lead to the case where $G<M$ resulting in negatively skewness. Similarly, the parameter $\nu=1 / C$ primarily controls the kurtosis.

\section{Acknowledgments}

The second author is a Fellow of the Fund for Scientific Research - Flanders (Belgium) (F.W.O. - Vlaanderen). The authors thank Jessica Cariboni for computational assistance and stimulating comments, Goldman Sachs for kindly providing CDS data, Geert Gielens and Jurgen Tistaert for fruitful discussions. 


\section{References}

[1] Bertoin, J. (1996) Lévy Processes. Cambridge Tracts in Mathematics 121, Cambridge University Press, Cambridge.

[2] Beirlant, J., Schoutens, W., and Segers, J. (2005) Mandelbrot's Extremisme. Wilmott Magazine, March 2005.

[3] Black, F. and Scholes, M. (1973) The pricing of options and corporate liabilities. Journal of Political Economy 81, 637-654.

[4] Black, F. and Cox, J. (1976) Valuing corporate securities: some effects on bond indenture provisions. Journal of Finance 31, 351-367.

[5] Carr, P., Geman, H., Madan, D.H. and Yor, M. (2003) Stochastic Volatility for Lévy Processes. Mathematical Finance 13, 345-382.

[6] Carr, P. and Madan, D. (1998) Option Valuation using the Fast Fourier Transform. Journal of Computational Finance 2, 61-73.

[7] Cariboni, J. and Schoutens, W. (2004) Pricing Credit Default Swaps under Lévy Models. UCS-report 200407, K.U.Leuven, Leuven.

[8] - (2002) CreditGrades ${ }^{\mathrm{TM}}$, Technial Document, RiskMetrics Group, Inc.

[9] Cherubini U., Luciano E. (2001) Bivariate option pricing with copulas, Applied Mathematical Finance, 8, 1-17.

[10] Cherubini, U., Luciano E. and Vecchiato, W. (2004) Copula methods in Finance. Wiley.

[11] Clark, P. (1973) A subordinated stochastic process model with finite variance for speculative prices. Econometrica 41, 135-156.

[12] Cox, J., Ingersoll, J. and Ross, S. (1985) A theory of the term structure of interest rates. Econometrica 53, 385-408.

[13] Devroye, L. (1986) Non-Uniform Random Variate Generation. Springer.

[14] Duffie D., and Lando D. (2001) Term structure of credit spreads with incomplete accounting information, Econometrica, 69, 633-664.

[15] Gregory, J., and Laurent, J.P.(2003) Basket Default Swaps, CDO's and Factor Copulas, mimeo, BNP Paribas

[16] Leland, H. (1994) Corporate debt value, bond convenants, and optimal capital structure. Journal of Finance 49, 1213-1252.

[17] Li, D. X. (2000) On default correlation: a copula function approach, Journal of Fixed Income, 9, 43-54.

[18] Longstaff, F. and Schwartz, E. (1995) A simple approach to valuing risky fixed and floating rate debtebt value, bond convenants, and optimal capital structure. Journal of Finance 50, 789-819.

[19] Madan D.B. (2000) Pricing the Risk of Default, University of Maryland, Working Paper.

[20] Madan, D.B., Carr, P. and Chang, E.C. (1998) The variance gamma process and option pricing. European Finance Review 2, 79-105.

[21] Madan, D.B. and Milne, F. (1991) Option pricing with V.G. martingale components. Mathematical Finance $\mathbf{1}(4), 39-55$.

[22] Madan, D.B. and Seneta, E. (1987) Chebyshev polynomial approximations and characteristic function estimation. Journal of the Royal Statistical Society Series B 49(2), 163-169. 
[23] Madan, D.B. and Seneta, E. (1990) The v.g. model for share market returns. Journal of Business 63 , 511-524.

[24] Mandelbrot, B.B. and Taylor, H.M. (1967) On the distribution of stock price differences. Operations Res. 15, 1057-1062.

[25] Marshall, A.W. and Olkin, I. (1988) Families of multivariate distributions. Journal of the American Statitical Association, 83, 834-841.

[26] Merton, R. (1974) On the pricing of corporate debt: the risk structure of interest rates . Journal of Finance 29, 449-470.

[27] Nelsen R.B. (1999) An introduction to copulas, Lectures Notes in Statistics, Springer-Verlag, New York.

[28] Sato, K. (2000) Lévy Processes and Infinitely Divisible Distributions. Cambridge Studies in Advanced Mathematics 68. Cambridge University Press, Cambridge.

[29] Schönbucher, P. (2003) Credit Derivatives Pricing Models. Wiley.

[30] Schoutens, W. (2003) Lévy Processes in Finance: Pricing Financial Derivatives. Wiley.

[31] Zhou, C. (1997), A Jump-Diffusion Approach to Modeling Credit Risk and Valuing Defaultable Securities, working paper, Federal Reserve. 

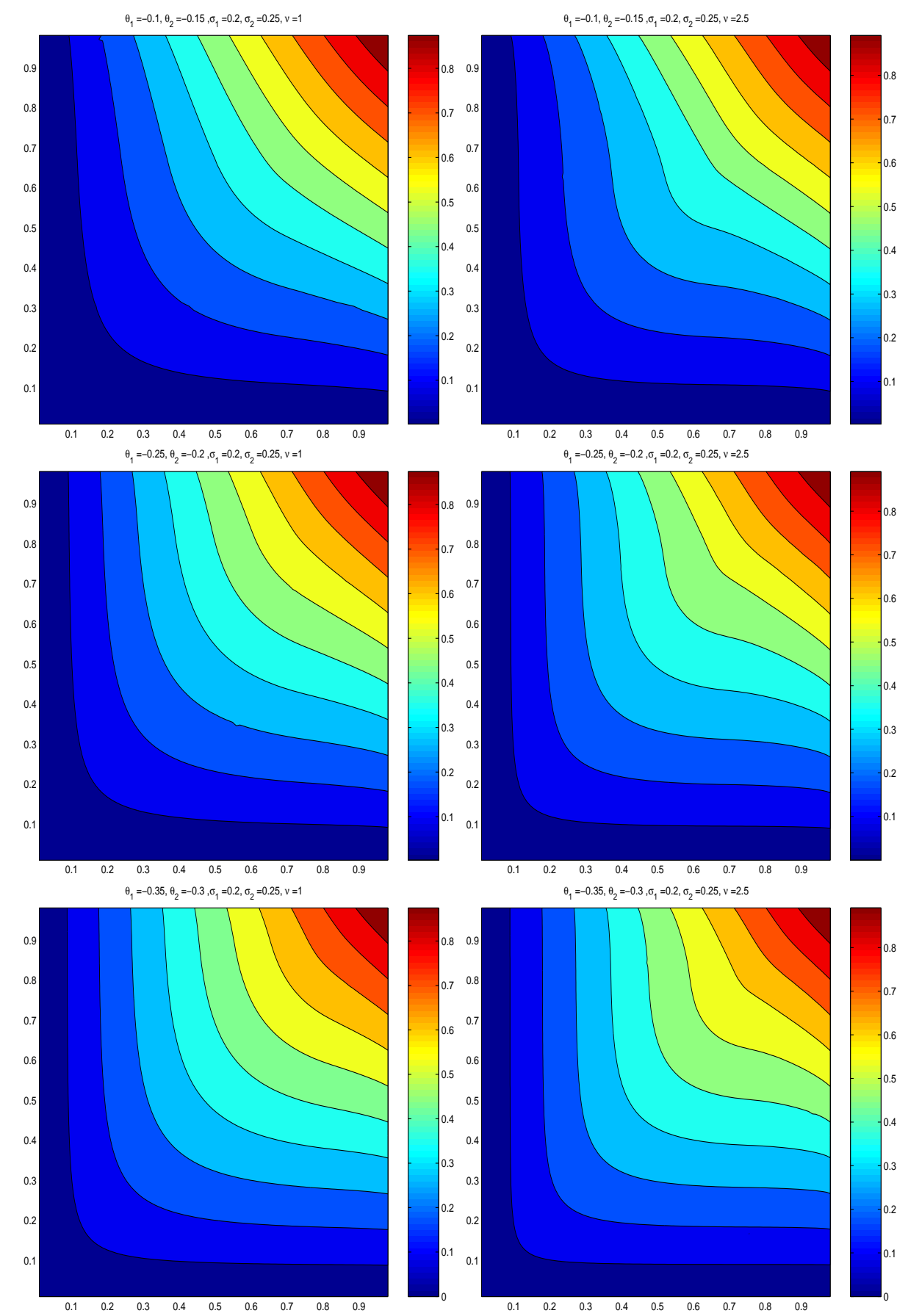

Figure 2: Contour plots of Copula for various parameter settings 

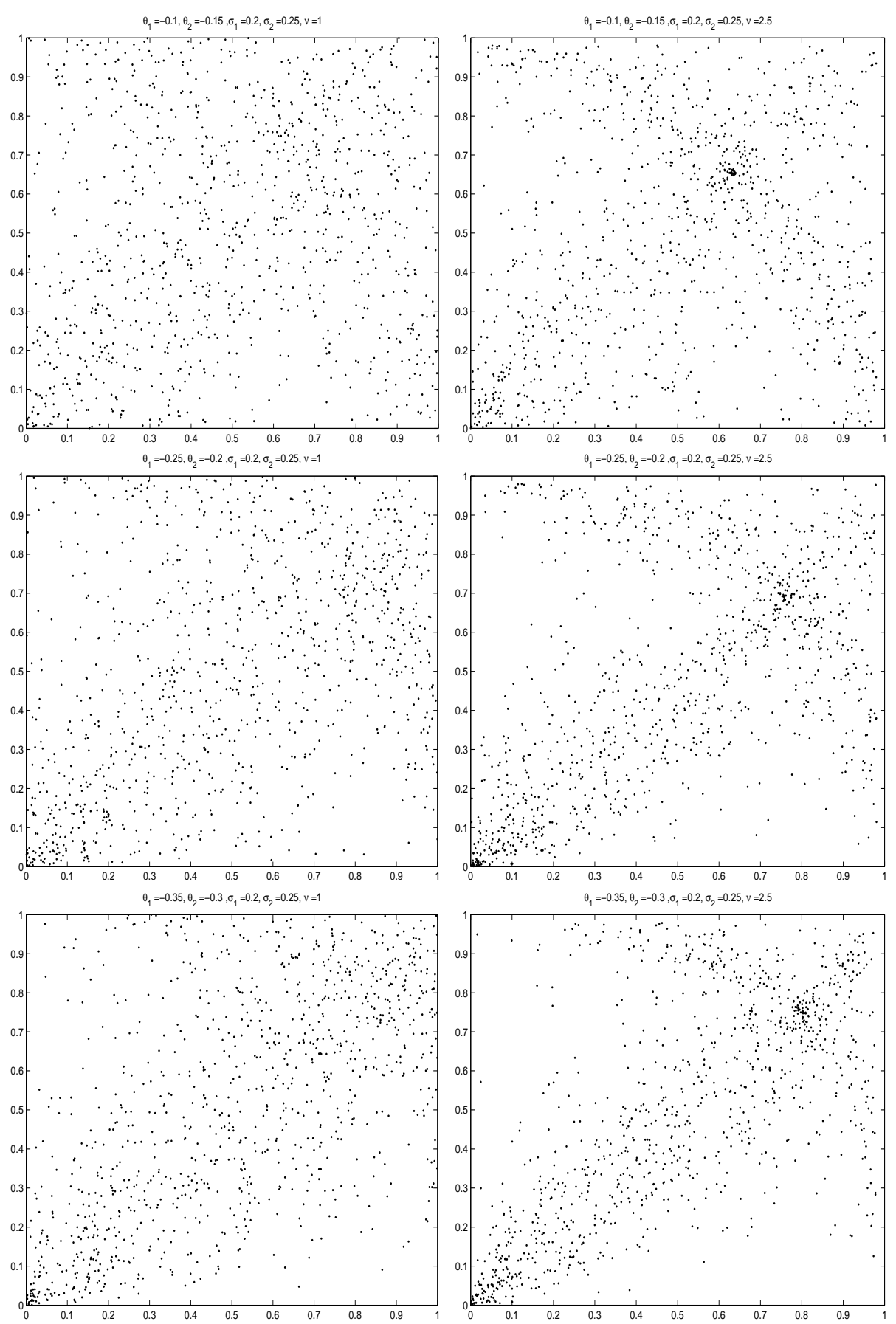

Figure 3: Scattered Plot of Copula for various parameter settings 

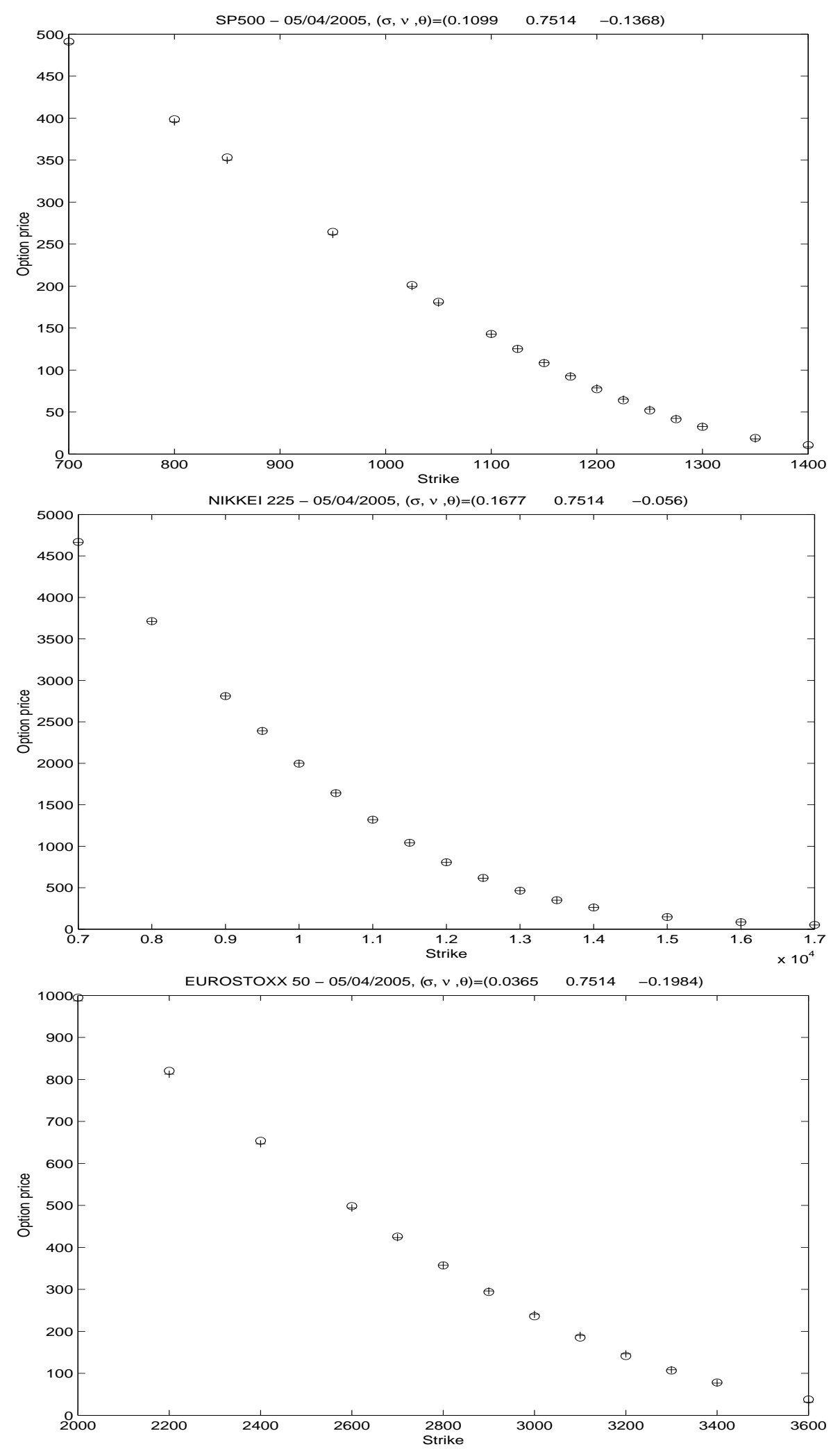

Figure 4: Multivariate Calibration of VG on Equity (Plus signs denote model prices, o-signs market prices). 

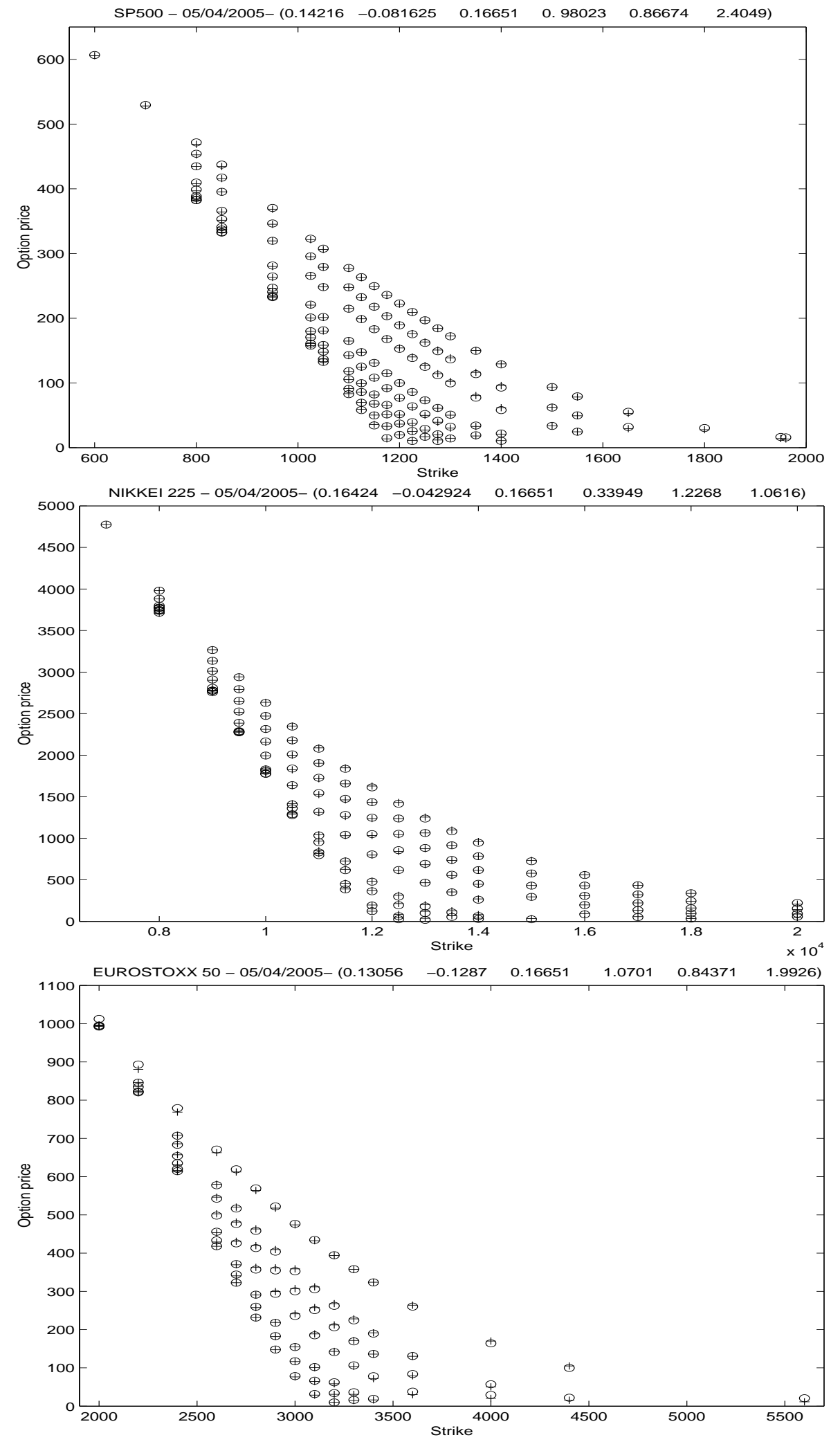

Figure 5: Multivariate Calibration of VG-SV on Equity (Plus signs denote model prices, o-signs market prices). 

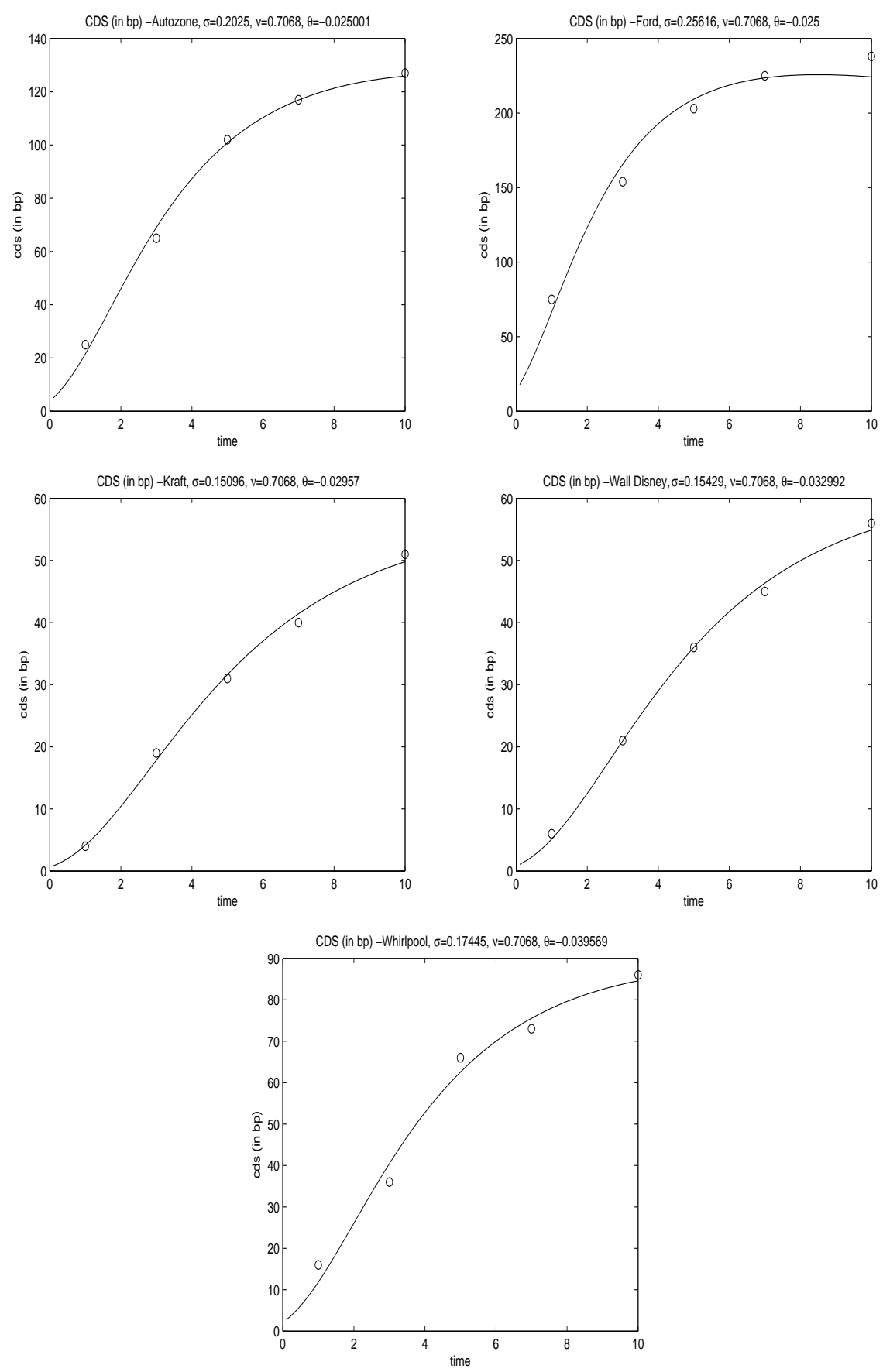

Figure 6: Multivariate Calibration 